\title{
Disease Milestone Instance Name
}

National Cancer Institute

\section{Source}

National Cancer Institute. Disease Milestone Instance Name. NCI Thesaurus. Code

C162265.

The name of a specific event or activity that can be anticipated in the course of a disease, but whose timing is not controlled by the study schedule. 